\title{
Tangence
}

\section{Livres pour tous : le flou des frontières entre fiction pour enfants et fiction pour adultes 1}

\section{Sandra L. Beckett}

Numéro 67, automne 2001

L'écriture pour la jeunesse : de la production à la réception

URI : https://id.erudit.org/iderudit/009612ar

DOI : https://doi.org/10.7202/009612ar

Aller au sommaire du numéro

Éditeur(s)

Presses de l'Université du Québec

ISSN

0226-9554 (imprimé)

1710-0305 (numérique)

Découvrir la revue

Citer cet article

Beckett, S. L. (2001). Livres pour tous : le flou des frontières entre fiction pour enfants et fiction pour adultes1. Tangence, (67), 9-22.

https://doi.org/10.7202/009612ar
Résumé de l'article

Résumé

L'effondrement des frontières entre textes pour enfants et textes pour adultes est un phénomène très répandu dans les littératures occidentales

contemporaines. Au cours des dernières années, on a vu un véritable afflux d'auteurs franchir, dans les deux sens, la frontière entre fiction pour adultes et fiction pour enfants. En s'appuyant sur un choix de romans et d'albums parus dans de nombreux pays, cette étude examine la tendance con- temporaine d'une littérature destinée à un double lectorat. 


\title{
Livres pour tous: le flou des frontières entre fiction pour enfants et fiction pour adultes ${ }^{1}$
}

\author{
Sandra L. Beckett, Brock University
}

Lá littérature destinée à un double lectorat n'est pas un phénomène nouveau, comme en témoignent de nombreux "classiques" de la littérature enfantine, des contes de fées à Alice au pays des merveilles et à Pinoccbio. Selon Bettina KümmerlingMeibauer, $76 \%$ des auteurs cités dans son ambitieuse encyclopédie, Klassiker der Kinder- und Jugendliteratur: Ein internationales Lexikon", sont, dans une certaine mesure, des "crosswriters ${ }^{3}$. Les best-sellers de Gallimard Jeunesse ne révèlent pas autre chose: Le Petit Prince d'Antoine de Saint-Exupéry, Vendredi ou La vie sauvage de Michel Tournier, Les contes du cbat perché de Marcel Aymé et L'enfant et la rivière d'Henri Bosco viennent en tête de liste. Le nombre d'auteurs célèbres qui ont écrit pour les enfants en France au $\mathrm{XX}^{\mathrm{e}}$ siècle est impressionnant: Michel Butor, Marguerite Duras, Jean Giono, Max Jacob, Eugène Ionesco, JeanMarie Le Clézio, Jacques Prévert, Claude Roy, Marguerite Yourcenar, etc. Au cours des dernières années, on a assisté, dans de nombreux pays, à un véritable afflux d'auteurs qui franchissent, dans les deux sens, la frontière entre fiction pour adultes et fiction pour enfants. Le phénomène que Peter van den Hoven décrit dans Grensverkeer (Circulation frontalière) au sujet de la littérature des Pays-Bas est, en réalité, un phénomène international qui se manifeste dans la plupart des littératures occidentales ${ }^{4}$. La

1. Des éléments de cette enquête sont repris dans un article, à paraître dans Perspectives contemporaines du roman pour la jeunesse (Actes du colloque de l'Institut international Charles Perrault, $1^{\text {er}}-2$ décembre 2000), Paris, L'Harmattan.

2. Bettina Kümmerling-Meibauer, Klassiker der Kinder- und Jugendliteratur: Ein internalionales Lexikon, 2 t., Stuttgart, Metzler, 1999.

3. Bettina Kümmerling-Meibauer, "Crosswriting as a Criterion for Canonicity: The Case of Erich Kästner", dans Sandra L. Beckett, Transcending Boundaries. Writing for a Dual Audience of Cbildren and Adults, New York, Garland, 1999, p. 25, note 11 .

4. Voir les essais réunis dans Sandra L. Beckett, Transcending Boundaries, ouvr. cité, où quatorze auteurs de huit pays évoquent des textes, à la fois romans et albums, appartenant à une vaste aire géographique et culturelle, du Canada aux Antilles, de la Russie à l'Australie. 
diversité des chemins, parfois tortueux, que prennent ces livres pour arriver à s'adresser aux deux publics est remarquable, mais l'on est surtout frappé par le nombre croissant de livres qui se destinent, d'entrée de jeu, aux lecteurs de tout âge.

\section{Chassés-croisés éditoriaux}

Le statut des textes qui enchantent un double public d'enfants et d'adultes reste souvent très ambivalent. Ainsi, la plupart des critiques et des lecteurs semblent avoir rangé à tout jamais l'auteur norvégien Tormod Haugen parmi les écrivains pour la jeunesse. Quand son roman Romanen om Merkel Hanssen og Donna Winter og den store flukten (L'bistoire de Merkel Hanssen et Donna Winter et leur grande évasion, 1986) a paru dans une collection pour adultes, les critiques l'ont traité en tant que livre pour la jeunesse. À l'inverse, rares sont ceux d'entre eux qui ont émis des réserves quand le complexe Skriket fra jungelen (Le cri de la jungle, 1989) a été édité dans une collection jeunesse; ce roman a d'ailleurs valu à l'auteur la Médaille Andersen en 1990.

Certains ouvrages sont classés alternativement comme livres pour enfants et comme livres pour adultes. Les livres publiés pour un public dans un contexte peuvent paraitre pour un autre public dans un autre contexte, par exemple à une autre époque ou dans un autre pays ou une autre culture. Examinons le cas de l'auteur britannique Philip Pullman. Nortbern Lights (1995), le premier tome de sa trilogie His Dark Materials ( $A$ la crotsée des mondes) a reçu la Médaille Carnegie (1996), ainsi que plusieurs autres prix décernés aux livres de jeunesse en Angleterre. De la même façon, la version française, Les royaumes du Nord, et la suite, La tour des anges, ont paru chez Gallimard Jeunesse. Cependant, en Suède, le roman de Pullman est sorti dans une édition pour adultes. Aux États-Unis, il a été publié sous le titre The Golden Compass dans une collection pour adultes et a été élu le meilleur livre de l'année. Pullman lui-même m'a assuré qu'il n'avait jamais de lectorat particulier en vue. Northern Lights a été publié en Angleterre comme un livre pour enfants parce que la maison Scholastic ne publie que des livres pour enfants, alors que l'éditeur américain, Knopf, a aussi un service pour adultes et peut donc vendre facilement aux deux publics. Si Pullman se félicite que le livre ait pu atteindre deux publics dans certains pays (par exemple en Allemagne et au Danemark), il avoue n'être pour rien dans cette déci- 
sion. Par ailleurs, l'auteur insiste sur le rôle consiclérable que joue l'illustration qui apparait sur la jaquette. La belle illustration d'Eric Rohmann, qui figure sur la jaquette de l'édition américaine, a été reprise avec succès dans de nombreux autres pays parce qu'elle s'adresse aussi bien aux adultes qu'aux enfants, tandis que la jaquette hongroise, qui représente une enfant de six ans vêtue d'un gilet de laine rose, en train de pleurer (choix "grotesquement impropre", selon l'auteur "), interdit la possibilité même d'un double public. Le troisième tome de la série, attendu avec impatience dans de nombreux pays, est paru à la fin de l'année 2000 .

L'un des grands phénomènes littéraires des toutes dernières années est la série "Harry Potter" de J. K. Rowling. Lors de la conception de la série, Rowling n'a pas considéré son public éventuel. Elle insiste sur le fait qu'elle n'a jamais pensé à écrire pour les enfants et que c'est plutôt la littérature de jeunesse qui l'a choisie $^{6}$. Le premier tome, Harry Potter and the Philosopher's Stone, est sorti dans une collection destinée aux enfants en Angleterre, mais il s'est hissé à toute allure au sommet de la liste des best-sellers pour adultes, de sorte que Bloomsbury n'a pas tardé à en publier une édition pour ce public inattendu. Les deux livres sont identiques, à l'exception de la couverture, qui est plus sobre pour la seconde édition ${ }^{7}$. Salué par des critiques dithyrambiques, le livre a reçu plusieurs prix importants (le National Book Award, le prix Smarties et le Children's Book Award); de plus, il a été sélectionné pour la Médaille Carnegie. Sous le titre Harry Potter and the Sorcerer's Stone, le livre a été publié pour les enfants aux ÉtatsUnis, où l'on a vu se répéter le même prodigieux phénomène. Il est resté trente-huit semaines en tête de la liste des best-sellers du New York Times. En France, Harry Potter à l'école des sorciers et les suites, Harry Potter et la chambre des secrets et Harry Potter et le prisonnier d'Azkaban, ont paru chez Gallimard Jeunesse.

Les deuxième et troisième tomes ont été couronnés du même succès que le premier. Dès sa parution, Harry Potter and the

5. Courriel de l'auteur, 5 mai 2000.

6. Voir "Flying Starts. Seven first-time children's authors and illustrators talk about their fall debuts" (21 décembre 1998).

7. Un collègue suédois qui a commandê chez Amazon, en Grande-Bretagne, les trois tomes alors publiês a reçu le premier dans une édition pour adultes, avec sa couverture sobre et grise, et les deux autres dans une édition pour enfants. 
Chamber of Secrets a été le numéro un de la liste des best-sellers en Angleterre, dépassant les ventes d'auteurs tels que Jeffrey Archer et John Grisham. Le troisième tome a été sélectionné pour le prestigieux prix Booker et, plusieurs mois avant sa parution, en juillet 2000 , le quatrième était le best-seller de la liste d'Amazon. com! La maison Scholastic n'arrive pas à satisfaire la demande qu'a créée cet engouement pour Harry Potter. Le chiffre des ventes est stupéfiant: en plus des millions d'exemplaires déjà imprimés, plusieurs autres millions d'exemplaires sont d'ores et déjà commandés. Le réalisateur du film Harry Potter, chez Warner, est Chris Columbus, lequel a réalisé de nombreux films destinés à un public familial, tels que Home Alone. La compagnie qui fabrique les cartes Pokemon a annoncé des cartes Harry Potter ${ }^{8}$, et Warner a signé un contrat avec Mattel pour sortir des jouets. Le jeune sorcier a même orné la couverture de Time en 1999!

Un autre best-seller international, Sofies verden (Le monde de Sophie) de Jostein Gaarder, a paru en Norvège et dans la plupart des pays européens comme un livre pour les adolescents, selon l'intention de l'auteur; mais aux Etats-Unis, il a été publié pour les adultes. J'ai découvert le livre en 1995 au Salon du livre de Paris, où une foule de Français, pour la plupart des adultes, faisait la queue devant le stand où l'auteur signait l'édition française du Monde de Sophie, parue chez Seuil Jeunesse. En France, le roman a été publié par la suite dans une collection pour adultes. De même, les ouvrages ultérieurs de Gaarder, Le mystère de la patience et Dans un miroir obscur, ont paru à la fois chez Seuil Jeunesse et dans une édition pour adultes. Il arrive parfois qu'un même roman soit publié simultanément dans une collection pour adultes et une collection pour la jeunesse: ce fut le cas pour Maina de Dominique Demers en 1997.

Le texte d'un album peut s'adresser ultérieurement aux adultes dans un tout autre contexte. Michel Tournier affirme que son meilleur livre est Pierrot ou les secrets de la nuit (1979), publié dans une édition pour enfants en 1979 et couronné par le prix du meilleur livre étranger à la foire de Leipzig en 1981. Dix ans après sa parution en album, Pierrot a été repris dans Le médianocbe amoureux, pour adultes et, quelques mois plus tard, dans Les

8. Il s'agit des Wizards of the Coast, société appartenant à Hasbro et dont le centre d'exploitation est à Seattle (The Seatlle Times, 12 février 2000). 
contes du médianoche en Folio Junior. De même, Angus, que Tournier a écrit en hommage à Victor Hugo après une relecture de "L'Aigle du casque", a paru en album avant d'être inséré dans les deux Médianocbe.

\section{Des adultes vers les enfants}

Si un certain nombre de livres d'abord publiés pour les enfants trouvent par la suite un public adulte, le phénomène inverse est beaucoup plus fréquent. De nombreux livres destinés aux adultes passent ultérieurement à l'édition pour la jeunesse sans aucune adaptation, les seuls changements étant d'ordre paratextuel. Dans les années qui ont suivi la création d'un service Jeunesse en 1978, Gallimard a repris à l'intention des enfants de nombreuses nouvelles de ses auteurs les plus connus ${ }^{9}:$ Les bottes de sept lienes et autres nouvelles de Marcel Aymé (1980), Notre-Dame des Hirondelles de Marguerite Yourcenar (1982), L'bomme qui plantait des arbres de Jean Giono (1983). Ce texte de Giono a connu, on le sait, beaucoup de succès auprès des enfants à partir de 1987 grâce au court métrage d'animation de Frédéric Back. "La fugue du petit Poucet" ainsi qu' Amandine " de Tournier ont paru d'abord dans le magazine Elle. Mais alors qu' Amandine" est ensuite sorti en album, avant de paraître dans Le coq de bruyère, "La fugue du petit Poucet. a suivi le chemin inverse, paraissant d'abord dans la collection "Contes et récits" pour adultes, puis en album. Les deux contes figurent également dans les Sept contes de Folio Junior et, plus récemment, ils ont été réédités dans Le miroir à deux faces (1994), un livre somptueusement illustré par Alain Gauthier, mais qui semble s'adresser autant, sinon davantage, aux adultes qu'aux enfants ${ }^{10}$.

Michel Tournier est un véritable maître de l'art du recyclage des textes; les siens franchissent les frontières dans les deux sens et ne cessent d'aller et venir, avec une facilité prodigieuse, entre les deux publics. En 1995, lors d'un entretien, l'auteur m'a confié qu'il pensait insérer le conte qui a donné son titre au Miroir à deux faces, paru d'abord dans une revue pour adultes, dans une

9. Certains poèmes ont subi la même transposition, par exemple ceux de Jacques Prévert publiés dans la collection "Folio Cadet".

10. Tournier lui-même m'a assuré que ces illustrations, à la différence de celles que Gauthier avait faites en 1979 pour La fugue du petit Poucet, lui semblaient peu convenir aux enfants (entretien du 6 juillet 1995). 
réécriture pour adultes de la couleuvrine, qui avait été commandée par la revue pour enfants, Je bouquine. Mais jusqu'à présent, le roman pour adultes n'a pas vu le jour. Au reste, ce recyclage fréquent de ses textes semble témoigner autant de la paresse de l'auteur que de son sens des affaires - et Tournier serait parmi les premiers à le reconnaître.

À l'exception de Voyage au pays des arbres, tous les titres de Le Clézio parus pour les jeunes ont d'abord été conçus à l'intention de lecteurs adultes. La plupart des titres (Lullaby, Celui qui n'avait jamais vu la mer, Peuple du ciel, Villa Aurore, La grande vie) sont tirés de Mondo et autres bistoires ou de La ronde et autres faits divers. Le plus récent, Pawana (1995), est un récit plus long sur les baleines, écrit en 1988 pour le théâtre et publié en 1992 pour les adultes. L'édition dans la collection "Lecture Junior ", illustrée par Georges Lemoine, semble avoir eu beaucoup plus de succès que l'édition originale pour adultes. Tout en niant l'existence de la littérature pour enfants, Le Clézio a produit, presque malgré lui, un corpus important de livres pour la jeunesse, parce qu'il croit fermement qu'un auteur "ne doit jamais perdre de vue qu'il s'adresse aussi " aux "enfants à la veille de l'adolescence" qui sont, pour lui, de "vrais lecteurs ${ }^{11}$.

\section{Réagencements textuels et réécritures}

Parfois, il arrive qu'un passage est extrait d'un roman pour adultes, puis doté d'un nouveau paratexte à l'intention des enfants. L'année même où Tournier a publié Gaspard, Melcbior et Baltba$z a r(1980)$, on en a tiré le conte oriental, "Barbedor", que Sangali raconte au roi Hérode et à ses invités, pour le publier en album dans la collection "Enfantimages". Le roman Désert de Le Clézio, publié également en 1980, contient, lui aussi, un conte oriental, "Balaabilou", qui, en 1985, a subi le même sort que "Barbedor". Bien que le procédé soit le même dans les deux cas, il semble que l'album Barbedor ait été l'idée de l'auteur, tandis que l'album Balaabilou est un choix d'éditeur ${ }^{12}$. À l'instar de son protagoniste éponyme, Barbedor ne cesse de renaître: le conte a été repris dans Sept contes de la collection Folio Junior en 1984 et, plus récem-

11. Voir Sandra L. Beckett, De grands romanciers écritent pour les enfants, Montréal/Grenoble, Presses de l'Université de Montréal/ELLLG, 1997, p. 296 et 299 . 
ment, dans la collection Folio Cadet. Le cas de Barbedor illustre deux tendances opposées de la littérature de jeunesse contemporaine: repris d'un roman pour adultes et publié pour les enfants par un auteur qui cherche à transcender les frontières entre les différentes catégories de lecteurs, le conte est ensuite diffusé par un éditeur en fonction de classes d'âge de plus en plus précises.

Tournier affirme avoir été très étonné d'apprendre que la seconde version de Vendredi était, selon les éditeurs, un livre pour enfants. Si l'auteur semblait voir à l'origine dans Vendredi ou La vie sauvage une version pour enfants, il affirme maintenant, sur un ton catégorique, que le second Vendredi est simplement une ceuvre meilleure qui s'adresse à tous. Puisque Tournier mesure le succès de tous ses livres à leur réception par un enfant de douze ans, il ne partage pas toujours le point de vue de son éditeur en ce qui concerne leur public éventuel. En 1995, il m'a montré le manuscrit de la "version pour enfants" d'u un western religieux" qu'il comptait réécrire par la suite pour les adultes ${ }^{13}$, mais Eléazar ou La source et le buisson a paru en 1996 dans la célèbre "Collection blanche" de Gallimard. Tournier a toutefois dédié le court récit à la jeune fille de douze ans qui représente, selon lui, la lectrice idéale.

C'est l'éditeur qui avait demandé à Joy Kogawa de tirer un livre pour enfants du roman Obasan (1981), qu'elle avait écrit pour sensibiliser les Canadiens à la situation difficile qu'avaient éprouvée leurs concitoyens d'origine japonaise durant la Seconde Guerre mondiale. Cet ouvrage lui avait valu de nombreux prix, qu'il s'agisse du Books in Canada First Novel Award ou encore du Canadian Authors'Association Book of the Year Award ${ }^{14}$. La réécriture pour enfants, intitulée Naomis Road, est le seul récit que Kogawa ait destiné à la jeunesse, bien qu'elle reconnaisse n'avoir jamais écrit avec autant de facilité et se demande si elle ne devrait pas écrire plus de livres pour enfants ${ }^{15}$. Comme Vendredi

12. Sandra L. Beckett, De grands romanciers écrivent pour les enfants, ouvr. cité, p. 281 et 295.

13. Entretien inédit avec l'auteur, juillet 1995.

14. Voir Larissa Klein Tumanov, Between Literary Systems, Authors of Literature for Adults Write for Children, thèse de doctorat, University of Alberta, Edmonton, automne 1999, p. 18.

15. Kathleen Donahue, "Free-Falling" and "Serendipity": An Interview with Joy Kogawa ", Canadian Cbildren's Literature/Littérature canadienne pour la jeunesse (CCULCJ), Guelph (Ontario), no 84, 1996, p. 41-42. 
ou La vie sauvage ou le deuxième Comment Wang-Fô fut sauvé de Yourcenar, Naomi's Road est une réécriture pour les jeunes d'un texte destiné aux adultes, mais il a aussi connu une métamorphose supplémentaire, puisque le livre publié au Japon est un mélange issu de la version pour adultes et de celle pour enfants. Selon l'auteur, qui a répondu aux exigences d'un éditeur qui ne voulait pas se contenter d'une simple traduction en japonais de Naomi's Road, la troisième version conviendrait aux adolescents. My Place (1987) de l'Australienne aborigène Sally Morgan constitue un autre exemple de livre pour adultes qui a donné lieu à plus d'un ouvrage pour la jeunesse. En démêlant les fils enchevêtrés des récits entrelacés et complexes de son autobiographie, Morgan en a fait deux livres abrégés pour enfants ainsi qu'un album un peu plus cher, également intitulé My Place.

\section{Un double lectorat explicite}

Au cours des années 1990 , on a observé la création de collections pour les lecteurs de tout âge qui affichent clairement leur intention de s'adresser à un double lectorat. Ainsi la collection "16/96", créée à Montréal par La courte échelle en 1996 et dont les premiers titres étaient signés par des auteurs pour la jeunesse bien connus au Québec, s'adresse aux adolescents aussi bien qu'aux adultes. Six ans plus tôt, la maison d'édition espagnole Siruela, convaincue "qu'aucune frontière claire n'existe entre les titres pour enfants et ceux pour adultes ${ }^{16}$ ", avait lancé une collection intitulée "Las tres edades" pour les lecteurs de "huit à quatrevingt-huit ans". Contrairement à La courte échelle, Siruela a fait appel à de nombreux auteurs qui écrivaient plutôt pour les adul-

16. Bien que la nouvelle collection de Siruela soit destinée surtout aux enfants, on veut persuader ses lecteurs que "la bonne littérature ne connaît pas d'âge . On pense à la création de la "Bibliothèque blanche" par Gallimard dans les années 1950 pour permettre la formation, "à côté de la bibliothèque des parents", d'une bibliothèque des enfants composée "de textes d'une valeur littéraire certaine.. Gallimard a délibérément décidé de ne pas avoir recours a à des spécialistes de la littérature enfantine ", mais de faire appel à des écrivains "pour grandes personnes", à des "auteurs de livres sérieux". L'un des premiers titres choisis pour lancer la collection a été L'Enfant et la rivière d'Henri Bosco, qui avait gagné le Prix Théophraste-Renaudot en 1945 pour Le Mas Théotime. Voir "Bibliothèque blanche", Bulletin de la NRF, Paris, n" 75 , novembre 1953 , p. 16 ; Jacques Lemarchand, "Livres pour enfants", Bulletin de la NRF, $\mathrm{n}^{\circ} 144$, décembre 1950, p. 13b-14a. 
tes. L'un des premiers titres publiés dans la collection a été Caperucita en Manbattan de Carmen Martín Gaite, qui avait gagné le prestigieux Premio Nacional de Literatura en 1978 pour son roman, El cuarto de aträs. Bien que Martín Gaite ait déjà publié deux livres pour un jeune public, elle avoue n'avoir jamais eu l'intention d'écrire de livres pour enfants. Comme Tournier et tant d'autres auteurs qui sont publiés avec le même succès pour les adultes et les enfants, Martín Gaite résiste à la tentation de tracer une frontière distincte entre les deux lectorats. Elle fait remarquer que Cuentos completos, recueil de nouvelles pour adultes, plaît aux enfants de douze ans, tandis qu'à l'inverse, son "livre pour enfants", El castillo de las tres murallas, est apprécié par un public adulte et considéré par certains critiques comme sa meilleure cuvre ${ }^{17}$. Elle regrette que les préjugés qui prévalent contre la littérature de jeunesse en Espagne empêchent ce qu'elle appelle "un excellent roman "d'être considéré comme autre chose qu'une ceuvre mineure d'une grande romancière quion a priée un jour d'écrire pour les enfants. L'auteure était déterminée à ce que Caperucita en Manbattan ne partage pas le même sort que les deux livres antérieurs qui étaient destinés à rester à tout jamais des contes pour enfants. C'est ainsi que Caperacita en Manhattan, une réécriture du Petit Chaperon rouge sous forme de roman, avec treize illustrations de l'auteure, est devenue l'une des plus grandes réussites de la collection "Las tres edades "

On a tendance à classer un ouvrage illustré, aussi long et complexe soit-il, parmi les livres pour la jeunesse. L'auteur hollandais Wim Hofman a gagné et le Crayon d'Or et le prestigieux Prix Woutertje Pieterse en 1998 pour Zwart als inkt is bet verbaal van Sneeuwwitje en de zeven dwengen (Noire comme l'encre est l'bistoire de Blanche-Neige et les sept nains), un long texte très poétique illustré en noir, blanc et rouge par l'auteur. Le conte de Blanche-Neige devient l'objet d'un examen psychologique approfondi à la faveur d'une réécriture originale et controversée qui explore le côté cauchemardesque de l'enfance. L'auteur essaie d'imaginer la vie de cette petite enfant abandonnée dans la forêt et obligée de passer de longues années avec sept petits hommes bizarres, qui sont autant d'adultes autoritaires et préoccupés de leurs propres affaires. La petite fille meurt d'ennui et passe son

17. Carmen Martín Gaite, "Entrevista : Carmen Martin Gaite", Cuademos de fiteratura Infantil y Juvenal (CLIJ), Barcelone, n'26, mars 1991, p. 7. 
temps à écrire des lettres à sa mère, à Dieu, à la table, à la bougie. Dans cette version, lorsqu'elle prend la pomme empoisonnée, Blanche-Neige est pleinement consciente des conséquences. Elle veut mourir. Hofman avoue qu'il est question du suicide; c'est le sens des ratures d'encre noire qui défigurent le dessin de la petite fille sur la couverture ${ }^{18}$. Zwart als inkt est, en effet, une histoire très noire. L'épigraphe tirée de "Little Gidding " de T. S. Eliot ( What we call the beginning is often the end/And to make an end is to make a beginning n) annonce la scène finale, où c'est au tour de Blanche-Neige de regarder dans le miroir. Quand le livre a gagné deux prix importants de la littérature de jeunesse aux Pays-Bas, il a provoqué beaucoup de discussions au sujet du public visé, mais Hofman lui-même insiste sur le fait qu'il n'écrit pas pour un public en particulier.

En proposant ses réflexions sur les sortes de livres que la jeunesse continuera à lire à l'âge électronique, lors d'un forum que j'ai organisé pour la Modern Language Association (MLA) en 1997 dans le but d'examiner l'état de la littérature de jeunesse au seuil du nouveau millénaire, Wendy Lamb, directrice du service jeunesse de Bantam Doubleday Dell, a mentionné non seulement les textes interactifs, mais les albums sophistiqués qui plaisent aux enfants comme aux grandes personnes. Bien que l'album soit généralement considéré comme un genre exclusivement réservé aux enfants, pour beaucoup d'auteurs et d'illustrateurs contemporains, il constitue plutôt une forme narrative qui peut s'adresser aux lecteurs de tout âge. Aujourd'hui, les innovations graphiques et le dialogue souvent complexe entre texte et image invitent à des lectures à des niveaux différents par des lecteurs de tout âge. Comme Carole Scott l'affirme avec justesse, les enfants et les adultes sont souvent presque à égalité devant un album: parfois, la naïveté des enfants leur donne même un avantage sur les adultes parce qu'ils se sentent moins contraints par les conventions de la lecture et peuvent répondre en toute liberté aux ouvrages qui ne se conforment pas aux codes habituels ${ }^{19}$.

Sur la jaquette de The Stinky Cbeese Man and Otber Fairly Stupid Tales (Le petit bomme de fromage et autres contes trop faits) de Jon Scieszka et Lane Smith, figure cette mention que beau-

18. Entretien avec l'auteur, 5 avril 2000.

19. Carole Scott, "Dual Audience in Picturebooks", dans Sandra L. Beckett, Transcending Boundaries, ouvr. cité, p. 101. 
coup d'albums contemporains pourraient reprendre à leur compte: "Ages: All. Même si on y insiste sur le fait que la reliure a été renforcée, de manière à suggérer que le livre est assez solide pour résister aux mauvais traitements que pourraient lui infliger les tout-petits, cet album annonce clairement qu'il s'adresse à la fois aux enfants et aux grandes personnes. Dans cette réécriture désopilante de contes archi-connus, Scieszka joue avec les codes du genre qu'il parodie, par exemple dans "L'histoire du géant" où il intervertit "Il y avait une fois", qui est l'incipit, et "La fin". Le jeu métalittéraire est assez raffiné dans cet album qui expose constamment son statut de fiction. L'auteur joue de même avec les conventions du monde de l'édition et, en adaptant la rhétorique de la publicité, il insiste sur la fonction du livre dans notre société de consommation ${ }^{20}$. Cet album, qui a recours aux techniques du bricolage et du collage, peut répondre à tous les goûts, que l'on songe au texte ou aux illustrations. L'illustration qui accompagne "L'histoire du géant " juxtapose des dessins d'enfants exécutés avec des crayons de couleur et des images publicitaires, des illustrations tirées de livres pour la jeunesse récents et d'autres, reconnaissables uniquement par les adultes, tirées de vieilles éditions de classiques. Le mode irrévérencieux et parodique de cet album nous rappelle les Simpsons, qui séduisent également un public de tout âge.

$A$ Visit to William Blake's Inn de Nancy Willard annonce l'intention de l'auteure de s'adresser à un double lectorat dès le sous-titre "Poems For Innocent and Experienced Travelers". En 1981, ce livre de poèmes sur la vie dans une auberge imaginaire tenue par William Blake, et dont le personnel se compose de deux dragons, deux anges et un lapin, a étê le premier livre de poésie à gagner la Médaille Newbery. L'auteure m'a dit en mars 2000 qu'elle l'avait écrit "pour tous les âges, dans l'esprit d'un conteur de village ". Les enfants "s'assoient au premier rang, les grands-parents au fond, et l'histoire s'adresse à tous". À sa connaissance, son auditeur le plus jeune a deux ans et sa lectrice la plus âgée est une nonagénaire dont la vue déclinante lui faisait désirer un livre de poésie imprimé en gros caractères. L'auteur m'a rappelé que Blake dit avoir écrit ses chansons heureuses "in

20. Roderick McGillis, "Ages: All": Readers, Texts, and Intertexts in The Stinky Cheese Man and Other Fairly Stupid Tales", dans Sandra L. Beckett, Transcending Boundaries, ouvr. cité, p. 121-122. 
a book that all may read", et qu'il nous dit aussi que "every child may joy to hear [them] ${ }^{21}$ ". Willard, qui est universitaire ainsi qu'auteure et illustratrice, continue à produire des livres originaux et pleins d'imagination qui franchissent les frontières entre les âges. Le texte de son album Pish Posh Said Hieronymous Bosch (1991) a été publié, sans les illustrations, dans une anthologie de littérature fantastique. Son clernier album, The Tale I Told Sasha, est un poème narratif qui a paru dans The Formalist, une revue littéraire qui ne publie pas de textes destinés à la jeunesse.

The Great Escape from City Zoo (1997) de l'auteur australien Tohby Riddle offre un excellent exemple d'album qui s'adresse à la fois aux tout-petits et aux grandes personnes. Sélectionné pour le Children's Book Council of Australia Picture Book of the Year Award, cet album, aux illustrations monochromes, raconte une histoire très simple (quatre animaux - un fourmilier, un éléphant, une tortue et un flamant - s'échappent d'un zoo, en quête d'une nouvelle vie) qui peut être appréciée par les petits enfants. Mais la notice publicitaire affirme avec raison que le livre rejoint toutes les classes d'âge et les allusions parodiques et subtiles à la culture populaire y sont autant de clins d'ceil aux lecteurs plus âgés. Cet auteur-illustrateur est aussi caricaturiste pour The Sydney Morning Herald et d'autres publications, comme nous l'apprend le texte figurant sur la jaquette. Une photographie de Riddle, représenté en compagnie des quatre évadés, est accompagnée d'une légende ironique: "Auteur, second à partir de la gauchen.

Le dernier ouvrage dont il sera question, Le petit chaperon rotge: une imagerie d'apres un conte de Charles Perrault de l'artiste suisse, Warja Lavater, est à la fois le plus ancien et le plus innovateur des ouvrages analysés. C'est le premier de six contes de Perrault que Lavater a transformés en des "livres-objets" quelle appelle des "imageries 22 ". Le livre se présente comne un accordéon de papier cartonné qui se déplie et le seul texte qu'on y retrouve apparaît dans la légende de la page de garde qui présente, au clébut, le code élémentaire à base de couleurs et de formes auquel recourt l'ouvrage. Le point rouge symbolise le Petit Chaperon rouge, le point noir symbolise le loup, plusieurs points

21. Lettre de lautcure, 21 mars 2000.

22. Bernadette Gromer, "Tête à tête: Entreticn avec Warja Lavater", La Revue des litres pour enfants. Paris, n"137-138, hiver 1991, p. 40-49 
verts représentent la forêt, etc. On serait peut-être tenté de classer d'emblée ce livre-objet, qui relève plutôt du jeu, comme un ouvrage pour la jeunesse. En réalité, l'imagerie a paru dans une édition de luxe, publiée d'abord par le Museum of Modern Art à New York et ensuite par Adrien Maeght, et se vendait donc dans les musées. Mais la littérature de jeunesse n’a pas tarcé à se l'approprier à l'intention des enfants qui n'avaient pas encore commencé l'apprentissage de la lecture. À l'Atelier des enfants du Centre Georges Pompidou, où on a présenté Le petit chaperon rouge en 1976, les enfants ont été fascinés par cet ouvrage insolite avec lequel ils pouvaient "jouer ${ }^{23}$. Depuis lors, les enseignants et les bibliothécaires ont confirmé que les imageries de Lavater séduisent les enfants de différents âges et de diverses cultures. Les remarques apparemment contradictoires de Lavater elle-même au sujet du public visé par les imageries suggèrent le statut ambivalent de celles-ci. Elle avoue avoir été étonnée lorsque les gens lui disaient, après la parution du Petit chaperon rouge, que leurs enfants l'aimaient et le comprenaient, mais, en 1993, elle affirme que ses imageries étaient "conçues pour les enfants". Si elle considère que les "pictogrammes pliés" sont autant de livres pour enfants dans la mesure où les signes du code visuel sont "faciles à comprendre et [...] ne nécessitent pas la connaissance d'un langage verbal particulier ${ }^{24}$, elle soutient ailleurs que La rose et la rainette est son seul livre "pour enfants". En fait, ces ceuvres originales transcendent les frontières et l'artiste reconnaît volontiers que "le code en pictogrammes" de ses imageries est lisible, "quels que soient l'époque, la nationalité et l'âge ${ }^{25}$. De nouveaux projets multimédias inspirés par ces ouvrages innovateurs cherchent à s'attirer de nouveaux acheteurs de tout âge. Une série de six films d'animation en images de synthèse a obtenu le premier prix Art Imagina 95. Actuellement, les imageries sont l'objet d'un projet cédérom interactif ${ }^{2 t}$.

23. Bernadette Gromer, "Tête à tête: Entretien avec Warja Lavater", art. cité, p. 40 .

24. Warja Lavater, "Perception: When Signs Start to Communicale", dans Ballis Shookman, The Faces of Physiognomy: Interdisciplinary Approacbes to Johann Caspar Lavater, Columbia (Caroline du Sud), Camden House, 1993, p. 186.

25. Warja Lavater, "Perception: When Signs Start to Communicate ", art cité. p. 186.

26. Voir le site suivant: http://www.ircam,fr/produits/techno/multimedia/ imageries-e. html. 


\section{Vers une littérature pour tous}

Les écrivains pour la jeunesse contemporains remettent en question les conventions, les codes et les normes qui ont traditionnellement régi le genre. Ils traitent de sujets auparavant tabous et ils utilisent, parfois avec plus d'audace que les auteurs qui restent du côté des adultes, des techniques narratives complexes (polyfocalisation, discours métafictif, mélange de genres, refus de clôture, intertextualité, ironie, parodie). Il n'est pas surprenant que les frontières entre fiction pour enfants et fiction pour adultes semblent devenir de plus en plus floues. Toutefois, on s'étonne du nombre et de la variété des livres (romans, albums avec peu de textes, livres sans aucun texte) qui transcendent ouvertement ces frontières pour s'adresser aux deux publics. Dans certaines langues, on a même inventé un nouveau mot pour désigner cette littérature, par exemple le terme allålderslitteratur en suédois. Ce qu'on appelle le crossover pbenomenon dans la culture anglo-saxonne est tellement répandu dans la littérature contemporaine de tant de pays qu'on serait tenté d'y voir l'un des attributs de l'âge post-postmoderne qu'annonce le nouveau siècle. 